\title{
Élaboration d'une boisson à partir d'écart de triage de dattes : clarification par traitement enzymatique et microfiltration
}

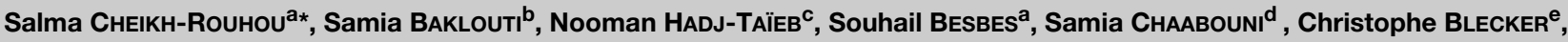
Hamadi ATtIA ${ }^{\mathrm{a}}$

\author{
a Unité analyses alimentaires, \\ École nationale d'ingénieurs de \\ Sfax, Route de Soukra, 3038, \\ Sfax, Tunisie, \\ salma_cr@yahoo.fr \\ b Lab. chimie industrielle I, \\ Dép. Génie des matériaux, \\ École nationale d'ingénieurs de \\ Sfax, Route de Soukra, 3038, \\ Sfax, Tunisie \\ ${ }^{c}$ Lab. génétique moléculaire \\ des eucaryotes, Centre de \\ biotechnologie, 3038 , \\ Sfax, Tunisie \\ d Unité enzymes et \\ bioconversion, École nationale \\ d'ingénieurs de Sfax, Route de \\ Soukra, 3038, Sfax, Tunisie \\ e Unité technologie des \\ industries agro-alimentaires, \\ Fac. Univ. Sci. Agron. \\ Gembloux, Passage des \\ Déportés 2, 5030 Gembloux, \\ Belgique
}

* Correspondance et tirés à part

Reçu le 9 février 2006 Accepté le 6 juin 2006

Fruits, 2006, vol. 61, p. 389-399 (c) 2006 Cirad/EDP Sciences All rights reserved

DOI: $10.1051 /$ fruits:2006038 www.edpsciences.org/fruits

RESUMEN ESPAÑOL, p. 399
Elaboration of a drink from date by-products: clarification by enzymatic treatment and microfiltration.

Abstract - Introduction. Despite their high nutritional value, by-products of dates are valueless in Tunisia, an important date-producing country. Our study aimed at testing a method for recovering these products by means of extraction, starting from a date paste, of a clarified drink. Materials and methods. The process of pulp extraction adopted was inspired by a traditional method. A rough solution obtained from the dilution of date paste was treated with enzymes (pectinases and cellulases) and by tangential microfiltration. The chemical composition, physico-chemical characteristics and rheological characteristics of the clarified drink obtained were determined. Results and discussion. The extracted rough solution has a high sugar content. The use of both microfiltration and enzymatic treatment resulted in a juice that has little acid content, with relatively low viscosity and turbidity and with a flow behaviour comparable to that of any other fruit juice. Conclusion. By-products of dates can be transformed, by the means of the usual processes, into a pleasant and marketable drink.

Tunisia / Pboenix dactylifera / dates / by-products / waste utilisation / transformation (of products)

\section{Élaboration d'une boisson à partir d'écart de triage de dattes : clarification par traitement enzymatique et microfiltration.}

Résumé - Introduction. En Tunisie, pays grand producteur de dattes, les écarts de triage de dattes restent non valorisés malgré une valeur nutritionnelle comparable à celle du fruit de haute valeur marchande. Notre étude a eu pour objectif de tester une voie de valorisation basée sur l'extraction, à partir d'une pâte de datte, d'une boisson de datte clarifiée. Matériel et méthodes. Le schéma technologique du procédé d'extraction de la pulpe a été inspiré d'un procédé traditionnel. Une solution brute obtenue de la dilution de pâte de dattes a été traitée par voie enzymatique (pectinases, cellulases) et par microfiltration tangentielle. La composition chimique, les caractéristiques physicochimiques et les caractéristiques rhéologiques de la boisson clarifiée obtenue ont été déterminées. Résultats et discussion. La solution brute extraite des dattes après broyage, dilution et filtration a une forte teneur en sucre. Le couplage des traitements enzymatique et de microfiltration a conduit à l'élaboration d'une boisson clarifiée, modérément acide, présentant une viscosité et une turbidité relativement faibles et ayant des caractéristiques d'écoulement comparables à celles des boissons et jus de fruits connus. Conclusion. Les écarts de triage de dattes peuvent constituer un sous-produit apte à être transformé, par le biais de procédés usuels, en une boisson agréable et commercialisable.

Tunisie / Pboenix dactylifera / datte / sous-produit / utilisation des déchets / transformation (des produits) 


\section{Introduction}

Dans le sud tunisien, le palmier dattier (Phoenix dactylifera L.) joue un rôle important tant sur le plan écologique que sur le plan socio-économique. La surface oasienne (22 500 ha) comprend environ $3 \mathrm{M}$ de dattiers dont la majorité (83\%) se trouve dans la région du Djérid et de Nefzawa, connue par la variété " Deglet Nour ".

Un grand tonnage de dattes matures (environ $30000 \mathrm{t}$ de dattes $\cdot \mathrm{an}^{-1}$ ) est perdu lors de la cueillette ou au cours du stockage, faute d'une présentation acceptable pour le consommateur. Ces dattes sont non com-

\section{Figure 1.}

Diagramme général de la fabrication d'une boisson de dattes clarifiée par un traitement enzymatique et une microfiltration (Tunisie).

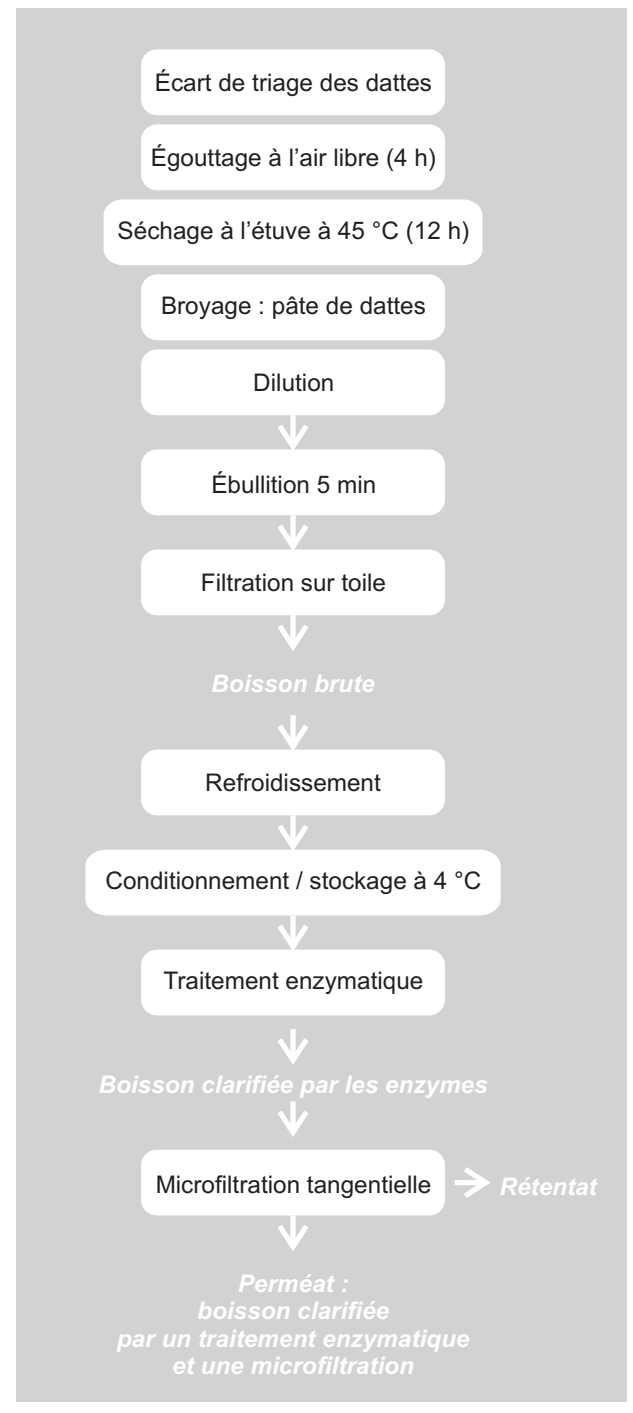

mercialisées et sont soit jetées, soit destinées à l'alimentation animale. Le rejet de ces sous-produits est une véritable perte économique puisqu'ils sont riches en éléments nobles pouvant être valorisés.

Les dattes présentent, en effet, des teneurs importantes en sucres [1], en éléments minéraux et en vitamines [2]. Elles contiennent également des protéines [1] et des lipides.

Les études de développement de nouveaux produits à partir des sous-produits de dattes sont à ce jour limitées. La littérature ne fait mention que de quelques travaux visant la formulation de nouveaux produits alimentaires comme, par exemple, la production de confitures, de gelées et de sirops de dattes [3-7] ou encore certaines transformations biochimiques telles que la production de biomasse à partir des dattes impropres à la consommation humaine [8-10] et la fermentation de pulpe de dattes en vue de la production de divers métabolites: acide citrique, oxytétracycline et éthanol [8, 11, 12].

En Tunisie, les écarts de triage de dattes provenant des industries de conditionnement et d'emballage sont essentiellement transformés en pâte de fruits. Cependant, les conditionneurs sont confrontés à des difficultés d'écoulement d'un tel produit dont les délais de conservation sont relativement courts [13]. Cela nous a conduits à prospecter une autre voie de valorisation de ces coproduits, celle de la production d'une boisson à base de dattes, via un procédé couplant un traitement enzymatique, à l'aide de pectinases et de cellulases, et une microfiltration tangentielle.

\section{Matériel et méthodes}

\subsection{Matière première}

Des dattes (Phoenix dactylifera L.) de la variété Deglet Nour ont été récupérées au stade "Tamr ", caractérisé par le ramollissement du fruit et par une couleur brunâtre, sur des palmiers des oasis de Deguech (Tunisie). Les fruits ont été maintenus au frais $\left(10^{\circ} \mathrm{C}\right)$ jusqu'au moment de leur transformation et/ou de leurs analyses. 


\section{2. Élaboration d'une boisson clarifiée à base de dattes}

\subsubsection{Fabrication de la boisson brute}

Le schéma adopté pour la fabrication de la boisson de dattes (figure 1) a été inspiré d'une préparation artisanale [6]. Après dénoyautage, les fruits ont été rincés à l'eau courante, puis égouttés pendant $4 \mathrm{~h}$ sur papier filtre avant d'être étuvés pendant $12 \mathrm{~h}$ à $45^{\circ} \mathrm{C}$

Une pâte de dattes a ensuite été obtenue par broyage. Elle a été additionnée d'eau (3 volumes d'eau : 1 volume de pâte), puis maintenue en ébullition pendant $5 \mathrm{~min}$ avant d'être filtrée sur une simple toile en textile. Le filtrat constituant la solution brute à base de datte a été transvasé dans des bocaux en verre à fermeture hermétique et conservé à $4{ }^{\circ} \mathrm{C}$ jusqu'au moment des analyses ou jusqu'au moment de la clarification enzymatique et/ou par membrane.

La turbidité de cette solution brute à base de datte s'est révélée relativement importante. La turbidité d'un jus de fruits dépend généralement de la teneur en pulpe dans le produit [14]. Elle est fonction de la composition et de la nature des polysaccharides (pectines, cellulose, hémicellulose), des protéines, des métaux, des tannins et des microorganismes qui y sont présents [15, 16]. Cette matière colloïdale contenue dans le jus aura tendance à floculer lentement [15] et contribuera, par conséquent, à augmenter la turbidité du produit. Cette turbidité se développera davantage après conditionnement et limitera la durée de conservation du produit. Pour cette raison, nous avons opté pour une clarification de la boisson de dattes brute élaborée par le biais d'un procédé enzymatique couplé à un procédé membranaire : la microfiltration tangentielle.

\subsubsection{Traitement enzymatique de la solution brute à base de datte}

Les préparations enzymatiques utilisées pour clarifier le filtrat extrait de la pâte de datte diluée ont été fournies par le laboratoire de génétique moléculaire des eucaryotes (Centre de biotechnologie, 3038, Sfax, Tunisie)
Deux préparations, présentant des températures et des $\mathrm{pH}$ optimaux identiques, ont été testées :

- une préparation à activité pectinase issue d'une souche de Penicillium occitanis, dont l'activité a pu être estimée à $60 \mathrm{UI} \cdot \mathrm{mL}^{-1}$;

- une préparation à activité cellulasique issue d'une souche de Penicillium pol 6. Cette préparation présente notamment une activité carboxyméthyl cellulasique $\left(3,7 \mathrm{UI} \cdot \mathrm{mL}^{-1}\right)$ et une activité $ß$-glucosidase sur le para-nitrophényl-ß-glucoside (pNPG) (275 Ul· $\left.\mathrm{mL}^{-1}\right)$.

Le traitement enzymatique de la solution brute à base de datte a été effectué à concentration de $2 \%$ (volume/volume), température de $50{ }^{\circ} \mathrm{C}$, agitation mécanique avec un agitateur à palettes $\left(100\right.$ tours $\left.\cdot \mathrm{min}^{-1}\right)$; il a duré $2 \mathrm{~h}$.

Pour suivre l'évolution de la clarification, un échantillon de $10 \mathrm{~mL}$ de la solution traitée a été prélevé à intervalles de temps réguliers, chauffé dans un bain-marie à $100{ }^{\circ} \mathrm{C}$ pendant $5 \mathrm{~min}$ afin d'inactiver les enzymes, puis centrifugé à 3000 tours $\cdot \mathrm{min}^{-1}$ pendant 5 min. Les surnageants récupérés, constituant la boisson traitée par les enzymes (BTE), ont été maintenus à $-20^{\circ} \mathrm{C}$ jusqu'au moment des analyses physico-chimiques. La turbidité et la viscosité de ces surnageants ont été mesurées immédiatement après la centrifugation car la congélation pourrait avoir un effet déstabilisant sur la fraction collö̈dale de la BTE.

\subsubsection{Microfiltration tangentielle}

Nos expérimentations ont été effectuées sur une installation de microfiltration classique [17]. La membrane utilisée a été de nature minérale (Carbosep), de configuration tubulaire avec des pores de 0,14 $\mu \mathrm{m}$ de diamètre moyen. La clarification de la boisson traitée par les enzymes a été effectuée à $40{ }^{\circ} \mathrm{C}, 150 \mathrm{kPa}$ de pression transmembranaire moyenne, $1,2 \mathrm{~m} \cdot \mathrm{s}^{-1}$ de vitesse tangentielle de circulation et à concentration volumique de facteur 1 . Le perméat récupéré a constitué une solution clarifiée par les enzymes et par microfiltration (BCEM). Il correspond à la boisson de datte élaborée. 


\subsection{Analyses physico-chimiques}

Le $\mathrm{pH}$, l'extrait sec total, l'extrait sec soluble et l'acidité titrable de la boisson élaborée ont été déterminés par des méthodes officielles [18] ; sa turbidité, exprimée en NTU (nephelometric turbidity unit), a été estimée par néphélométrie grâce à un turbidimètre de modèle Turb 555 IR ; la couleur a été évaluée par mesure de la densité optique à $420 \mathrm{~nm}$.

Le calcium, le magnésium, le potassium et le sodium ont été dosés par spectrométrie d'absorption atomique sur un appareil Hitachi Z6100 ; la teneur en protéines des dattes a été déterminée par la méthode de Kjeldahl en utilisant un facteur de conversion de 6,25 [18] ; les protéines contenues dans la boisson de datte et les perméats correspondants ont été dosées par la méthode de Bradford [19] en utilisant le réactif de Biorad.

Le glucose a été mesuré par la méthode à l'acide dinitrosalycilique (DNS) [20] ; les teneurs en fructose et en saccharose ont été estimées par HPLC sur un appareil Shimadzu 10 AT après clarification préalable par addition de ferrocyanure de potassium à $15 \%$ et de sulfate de zinc à $30 \%(\mathrm{p} / \mathrm{v})$.

La colonne utilisée a été du type échangeurs d'ions (polypore CA, $25 \mathrm{~cm}$, 4,6 mm de diamètre interne). La séparation a été conduite à $80^{\circ} \mathrm{C}$, à pression de 1000 psi (UI) et avec une vitesse de la phase d'élution de $0,3 \mathrm{~mL} \cdot \mathrm{m}^{-1}$. La détection a été assurée par réfractométrie différentielle (Shimadzu RID10A) et la quantification a été évaluée par comparaison des aires obtenues avec celles de standards (fructose, glucose et saccharose).

Les fibres totales ont été estimées par la méthode enzymatique-gravimétrique préconisée par Prosky et al. [21].

\subsection{Visualisation du colmatage}

L'induction du colmatage de la boisson de datte et de celle traitée par les enzymes en statique a été effectuée sur une membrane de même nature que celle utilisée dans les essais dynamiques et selon le protocole expérimental préconisé par Vétier et al. [22].

Les tronçons membranaires colmatés par le contact avec la boisson de dattes ont été observés par microscope électronique à balayage sur un appareil Philips XL30 après avoir subi une fixation chimique à la vapeur de glutaraldéhyde $(1 \%, \mathrm{v} / \mathrm{v})$ pendant $5 \mathrm{~min}$, un séchage au point critique du $\mathrm{CO}_{2}$ sur un appareil Baltec CPD 030 et une métallisation à l'or sur un appareil Baltec MED 20 [23].

\section{Résultats}

\subsection{Caractérisation de la matière première et de la solution brute obtenue à partir de dattes}

\subsubsection{Composition chimique}

La variété "Deglet Nour " est caractérisée par un extrait sec de l'ordre de $16 \%$, qui est légèrement supérieur à celui des variétés saoudiennes Birhi et Safri $(11,55 \%$ et $11,53 \%$, respectivement) [24]. Ce taux supérieur pourrait être dû à des facteurs environnementaux, au stade de maturation des dattes analysées et à certaines conditions de stockage.

Dans la pulpe de datte que nous avons étudiée, les sucres totaux (environ $88 \%$ de l'extrait sec) ont été prédominants, et de faibles teneurs en cendres et en matières azotées ont pu être notées (tableau I). Ces résultats sont comparables avec ceux rapportés dans la littérature pour d'autres variétés de dattes de qualité marchande [24-27]. L'altération sensorielle des dattes ne semble, donc, pas affecter leur valeur nutritionnelle. L'indice de qualité ou l'indice de dureté de la pulpe, défini comme étant le rapport entre les sucres totaux et la teneur en eau, a été de 5,38. Ce chiffre correspond à des dattes sèches pouvant être facilement conservées [27].

La fraction glucidique de la pulpe est formée essentiellement par le saccharose, le fructose et le glucose. Dans la variété Deglet Nour étudiée, c'est le saccharose qui a prédominé (tableau I) grâce, vraisemblablement, à une activité réduite de l'invertase [28]. La teneur en protéines se révèle relativement faible (2,5\%). Celles-ci présentent, cependant, une valeur biologique intéressante comparée à celle des protéines de l'ovalbumine [27]. 
Table I.

Comparaison des teneurs moyennes des principales composantes de dattes de la variété « Deglet Nour» et d'une boisson brute élaborée à partir du broyage de dattes dilué et filtré. Les valeurs données sont la moyenne de trois déterminations.

\begin{tabular}{|c|c|c|c|c|c|c|c|c|c|}
\hline \multirow[t]{2}{*}{$\begin{array}{l}\text { Produit } \\
\text { considéré }\end{array}$} & $\begin{array}{c}\text { Matière } \\
\text { sèche (ms) }\end{array}$ & Rendement & $\begin{array}{l}\text { Sucres } \\
\text { totaux }\end{array}$ & Saccharose & Glucose & Fructose & Cendres & Protéines & Fibres brutes \\
\hline & \multicolumn{2}{|c|}{$(\%)$} & \multicolumn{7}{|c|}{$\left(\mathrm{g} \cdot 100 \mathrm{~g}^{-1} \mathrm{~ms}\right)$} \\
\hline Pulpe de dattes & $83,60 \pm 0,15$ & - & $88,25 \pm 0,25$ & $57,59 \pm 0,12$ & $16,09 \pm 0,04$ & $14,56 \pm 0,09$ & $2,59 \pm 0,14$ & $2,51 \pm 0,17$ & $13,65 \pm 1,02$ \\
\hline $\begin{array}{l}\text { Boisson brute } \\
\text { de dattes }\end{array}$ & $21,45 \pm 0,92$ & $73,10 \pm 1,32$ & $82,50 \pm 0,20$ & $52,47 \pm 0,72$ & $15,86 \pm 0,35$ & $14,13 \pm 0,97$ & $2,40 \pm 0,60$ & $1,90 \pm 1,00$ & $3,82 \pm 0,83$ \\
\hline
\end{tabular}

(b) Teneur en macroéléments

\begin{tabular}{lcccc} 
Produit considéré & Potassium & Magnésium & Calcium & Sodium \\
\cline { 2 - 4 } & \multicolumn{4}{c}{$\left(\mathrm{mg} \cdot 100 \mathrm{~g}^{-1} \mathrm{~ms}\right)$} \\
Pulpe de dattes & $863,20 \pm 0,88$ & $41,66 \pm 0,29$ & $47,72 \pm 0,22$ & $10,24 \pm 0,12$ \\
Boisson brute de dattes & $859,90 \pm 0,05$ & $40,82 \pm 0,83$ & $47,61 \pm 0,01$ & $10,22 \pm 0,01$
\end{tabular}

La charge minérale de la pulpe est essentiellement dominée par le potassium [27].

Comme pour la matière première, la composition de la solution brute de datte a été dominée par la composante "sucre " (tableau I). Son degré Brix a été, en effet, de l'ordre de 22,5, résultat comparable à celui observé pour des boissons issues d'autres variétés de datte et extraites par le même procédé : $20,77^{\circ}$ Brix pour la variété Ruzeiz et $23,87^{\circ}$ Brix pour la variété Khudry [6]. Cependant, le degré Brix de la boisson élaborée à partir de dattes se révèle supérieur à celui du jus de pommes [(11,5 à 11,9) ${ }^{\circ}$ Brix] [26] et celui du jus de kiwi (12,6 ${ }^{\circ}$ Brix) [29].

Les teneurs en fibres et en protéines de la solution brute obtenue à partir de datte ont été relativement faibles. Comme pour la pulpe, le potassium a été l'élément dominant dans la boisson élaborée.

\subsubsection{Paramètres physico-chimiques}

L'analyse des paramètres physico-chimiques de la solution obtenue à partir de la pâte de datte (tableau II) montre que cette boisson est faiblement acide $(\mathrm{pH}$ 5,32)

\section{Table II.}

Caractéristiques d'une boisson brute élaborée à partir du broyage de dattes dilué et filtré. Les valeurs données sont la moyenne de trois déterminations.

\begin{tabular}{lcccc}
\hline $\begin{array}{l}\text { Solides solubles } \\
\left({ }^{\circ} \text { Brix }\right)\end{array}$ & $\mathrm{DO}_{420 \mathrm{~nm}}$ & $\mathrm{pH}$ & $\begin{array}{c}\text { Turbidité } \\
(\mathrm{NTU})\end{array}$ & $\begin{array}{c}\text { Acidité } \\
\left(\mathrm{mEq} \cdot \mathrm{L}^{-1}\right)\end{array}$ \\
\hline $22,50 \pm 0,20$ & $3,61 \pm 0,01$ & $5,32 \pm 0,01$ & $2230,30 \pm 1,00$ & $19,50 \pm 0,10$
\end{tabular}

comparée, par exemple, au jus de kiwi ( $\mathrm{pH}$ $3,58)$ [29]. Cependant, sa turbidité (2230 NTU) est environ sept fois plus élevée que celle de ce même jus de kiwi (299,5 NTU) [29] et cinq fois plus élevée que celle d'un jus de pomme traditionnel (440 NTU) [30]. Cette observation a motivé la clarification de la solution brute élaborée.

\subsection{Effet du traitement enzymatique sur la solution brute de datte}

Pour produire un jus clarifié de bonne qualité, il est nécessaire d'éliminer les matières en suspension, ce qui conduit à une meilleure clarté et homogénéité du produit, 
Figure 2.

Évolution de la turbidité d'une boisson, issue du broyage de dattes dilué, filtré et clarifié, en fonction du temps d'un traitement enzymatique par des cellulases, des pectinases et par un mélange de cellulases et de pectinases.

Figure 3.

Évolution de la viscosité d'une boisson, issue du broyage de dattes dilué, filtré et clarifié, en fonction du temps d'un traitement enzymatique par des cellulases, des pectinases et par un mélange de cellulases et de pectinases.
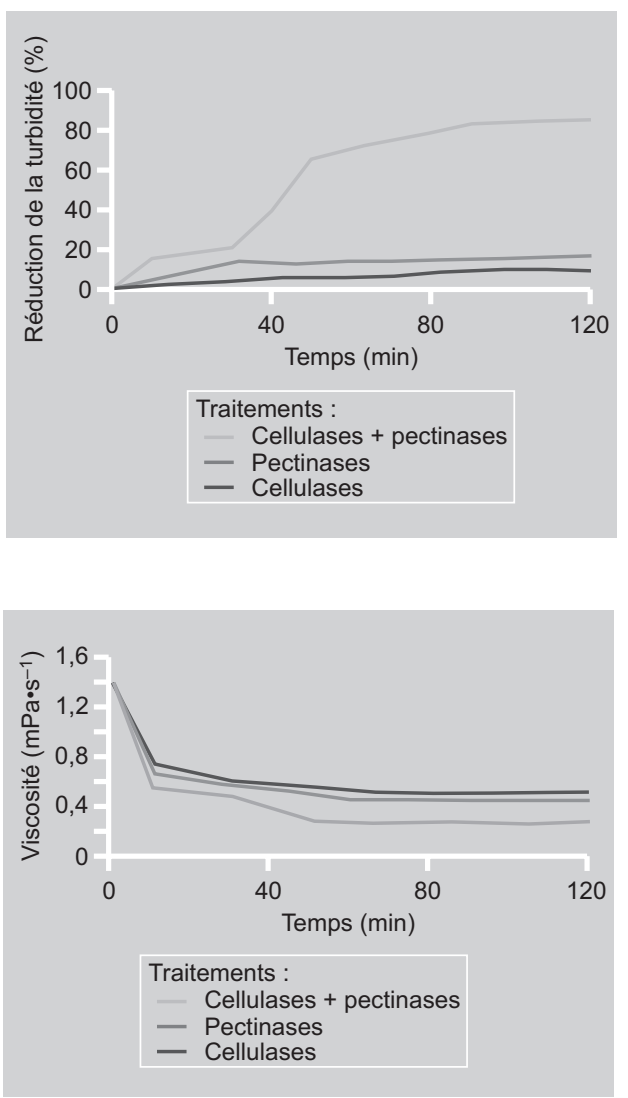

qui rendent compte de l'efficacité de clarification effectuée [31]. Les matières alors en suspension sont généralement des polysaccharides, des protéines, des tanins, des métaux et des microorganismes [32]. Le traitement enzymatique effectué avant filtration membranaire permet à la fois l'abaissement de la viscosité du jus et la réduction du contenu en solides solubles [32].

L'efficacité du traitement enzymatique de la solution brute obtenue à partir de pâte de dattes a été définie à l'aide des paramètres de turbidité et de viscosité dynamique [33].

\subsection{1. Évolution de la turbidité}

Pour réduire le taux de turbidité de la boisson brute de dattes, les pectinases sont apparues plus efficaces que les cellulases révélant, respectivement, $12 \%$ et $7 \%$ de réduction de la turbidité après $2 \mathrm{~h}$ de traitement (figure 2). Cependant, l'addition simultanée des deux types d'enzymes a eu un effet synergique puisque la turbidité a alors chuté, de $80 \%$.

\subsection{2. Évolution de la viscosité}

Le traitement de la boisson brute de dattes par le cocktail pectinases + cellulases a permis de réduire sa viscosité de $85 \%$ (figure 3). Cette réduction est moins marquée si la boisson est additionnée de pectinases seules ou de cellulases seules.

L'abaissement de la viscosité est dû à la solubilisation d'une grande partie des polysaccharides. Ainsi, les pectinases et les cellulases libèrent des monomères tels que l'acide galacturonique (cas de pectines) et des sucres simples (cas de cellulose). Les différentes hydrolyses ont donc engendré une moindre friction entre les constituants de la boisson et une meilleure affinité pour l'eau. Ces deux phénomènes induisent, l'un comme l'autre, une orientation facile de ces constituants de la boisson dans le sens de l'écoulement, il s'ensuit donc une plus faible viscosité. De même, les enzymes pectolytiques provoquent l'hydrolyse des molécules de pectines et la formation de complexes pectines-protéines qui précipitent [33]. La dégradation des pectines par les enzymes pectolytiques permet une réduction de la capacité de rétention d'eau et provoque une meilleure mobilité de la phase aqueuse [31], ce qui explique l'abaissement de la viscosité et de la turbidité observées pour la boisson de dattes traitée par le mélange de cellulases et de pectinases. Ce résultat nous a conduits à effectuer un traitement membranaire sur la boisson clarifiée par traitement enzymatique.

\subsection{Microfiltration tangentielle appliquée à la boisson clarifiée par voie enzymatique}

\subsection{1. Évolution de la performance}

Lors de la microfiltration tangentielle des solutions obtenues à partir de pâte de datte, la courbe présentant le flux de perméats au cours du temps a la forme typique du comportement du procédé de microfiltration tangentielle. Une première phase est caractérisée par une chute importante du flux due à la concentration de polarisation $[34,35]$ et 
une deuxième phase est caractérisée par la stabilisation du flux au bout de $7 \mathrm{~min}$ (figure 4).

Au début de l'opération de microfiltration, le flux de perméats de la solution clarifiée par traitement enzymatique $\left(130 \mathrm{~L} \cdot \mathrm{h}^{-1} \cdot \mathrm{m}^{-2}\right)$ est légèrement inférieur à celui rapporté pour du jus de pommes additionné de tannase $\left(200 \mathrm{~L} \cdot \mathrm{h}^{-1} \cdot \mathrm{m}^{-2}\right)$ [34] et pour celui du jus de melon prétraité avec un mélange de cellulases et d'hémicellulases [14] $\left(150 \mathrm{~L} \cdot \mathrm{h}^{-1} \cdot \mathrm{m}^{-2}\right)$. Ce flux relativement faible du jus de dattes est attribué à une moindre dégradation des pectines et de la cellulose par le mélange enzymatique pectinases + cellulases.

L'ajout d'enzymes avant la filtration membranaire a nettement amélioré le flux de perméats qui a été multiplié par un facteur 2 en passant de la solution brute non traitée à une solution clarifiée par traitement enzymatique pectinases + cellulases (figure 4). Cette augmentation du flux peut être expliquée par la réduction de la viscosité dynamique de la boisson brute (figure 3) qui induit un meilleur transfert de solvant à travers la lumière libre des pores [33, 36]. L'effet du colmatage externe serait par ailleurs plus aéré du fait de l'hydrolyse de la plus grande partie des fibres.

\subsubsection{Caractérisation des perméats}

\subsubsection{Composition chimique moyenne}

Comme constaté précédemment, la composition du perméat issu de la solution brute

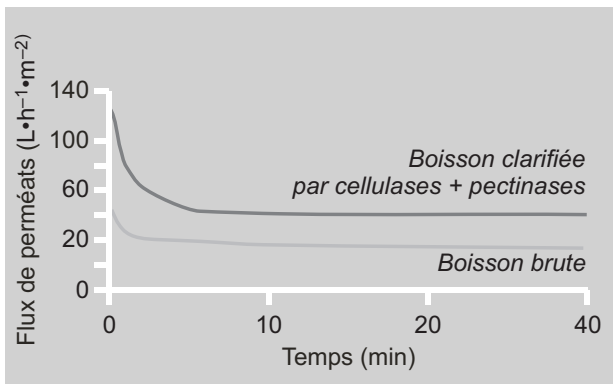

et celle du perméat issu de la solution clarifiée par un mélange de cellulases et de pectinases au cours de la microfiltration (tableau III) se sont stabilisées après 7 min de microfiltration tangentielle. Nous avons donc caractérisé les différentes fractions de ces perméats après 10 min de clarification par microfiltration.

Pour les deux types de solutions, brute ou prétraitée, les fractions glucidique, protéique et minérale ont traversé librement la lumière des pores de la membrane et sont passées en totalité dans le perméat. Ces fractions ont augmenté considérablement dans le perméat issu de la boisson clarifiée avec le mélange de pectinases + cellulases L'augmentation de la teneur en protéines doit être attribuée aux résidus enzymatiques [16].

\subsubsection{Paramètres caractéristiques}

L'effet de la microfiltration s'est traduit par un léger accroissement du pH dans les perméats récupérés : le pH est passé de 5,32 à 5,65 dans la solution brute et de 5,32 à 5,46
Figure 4.

Évolution du flux de perméats lors de la microfiltration d'une boisson, issue soit du seul broyage de dattes dilué et filtré, soit de cette même solution préalablement clarifiée par un mélange de cellulases et de pectinases. (Diamètre de pores moyen $=0,14 \mu \mathrm{m}$; facteur de concentration volumique $=1 ; \mathrm{T}=40^{\circ} \mathrm{C}$ et pression transmembranaire $=$ 1,5 bar.)

Table III.

Comparaison des compositions chimiques moyennes de perméats issus de la microfiltration d'une boisson brute élaborée soit à partir du seul broyage de dattes dilué et filtré, soit de cette même boisson préalablement clarifiée avec des enzymes.

\begin{tabular}{|c|c|c|c|c|c|}
\hline \multirow[t]{2}{*}{ Produit considéré } & \multirow[t]{2}{*}{$\begin{array}{c}\text { Matière sèche } \\
(\%)\end{array}$} & $\begin{array}{l}\text { Sucres } \\
\text { réducteurs }\end{array}$ & Protéines & \multirow[t]{2}{*}{$\begin{array}{l}\text { Fibres } \\
\text { brutes }\end{array}$} & Cendres \\
\hline & & \multicolumn{2}{|c|}{$\mathrm{g} \cdot 100 \mathrm{~g}^{-1}$ de matière sèche } & & $\mathrm{mg} \cdot 100 \mathrm{~g}^{-1}$ de matière sèche \\
\hline Perméat de la boisson brute & $17,74 \pm 0,50$ & $57,23 \pm 0,22$ & $1,45 \pm 0,10$ & Traces & $865,70 \pm 1,20$ \\
\hline $\begin{array}{l}\text { Perméat de la boisson clarifiée } \\
\text { avec le mélange cellulases et pectinases }\end{array}$ & $20,35 \pm 0,10$ & $120,32 \pm 0,07$ & $26,70 \pm 0,56$ & Traces & $825,45 \pm 2,55$ \\
\hline
\end{tabular}




\begin{tabular}{|c|c|c|c|c|}
\hline Produit considéré & $\mathrm{pH}$ & $\mathrm{DO}_{420}$ & ${ }^{\circ}$ Brix & $\begin{array}{l}\text { Turbidité } \\
\text { (NTU) }\end{array}$ \\
\hline Perméat de la boisson brute & $5,65 \pm 0,25$ & $0,43 \pm 0,11$ & $18,80 \pm 0,10$ & $3,50 \pm 0,25$ \\
\hline $\begin{array}{l}\text { Perméat de la boisson clarifiée } \\
\text { avec le mélange cellulases et pectinases }\end{array}$ & $5,46 \pm 0,12$ & $0,57 \pm 0,14$ & $24,00 \pm 0,20$ & $2,00 \pm 0,10$ \\
\hline
\end{tabular}

Température $=40^{\circ} \mathrm{C}$; pression transmembranaire $=1,5$ bar ; vitesse tangentielle de circulation $=$ $1,2 \mathrm{~m} \cdot \mathrm{s}^{-1} ;$ temps de filtration $=10 \mathrm{~min}$.

\section{Figure 5.}

Observations par microscope électronique à balayage du colmatage externe édifié en statique par contact soit avec une boisson issue du seul broyage de dattes dilué et filtré, soit de cette même solution clarifiée par un mélange de cellulases et de pectinases. dans la solution prétraitée. Par ailleurs, la clarification par microfiltration a entrainé une réduction spectaculaire de la turbidité des solutions : 2230 NTU mesurée pour la solution brute avant microfiltration à 3,5 NTU mesurée sur cette même solution

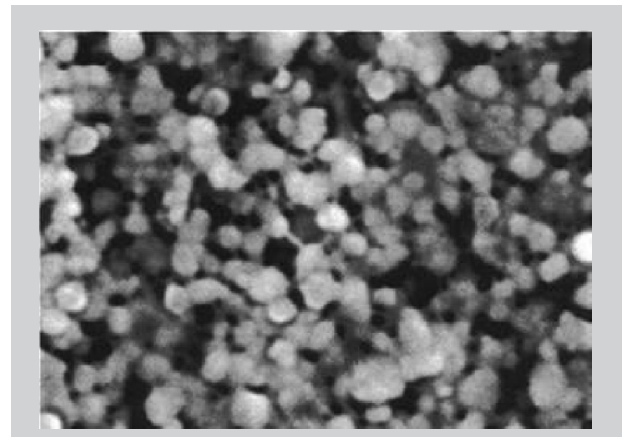

Boisson brute

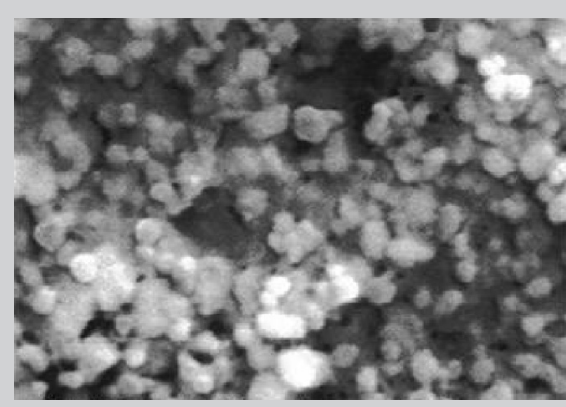

Boisson clarifiée par un mélange cellulases + pectinases

$500 \mathrm{~nm}$ après microfiltration ; et 2230 NTU avant microfiltration à 2 NTU après microfiltration pour la solution prétraitée avec un mélange d'enzymes (tableau IV). L'effet conjugué de l'activité enzymatique et de la microfiltration a permis une rétention des structures colloïdales, des fibres insolubles et d'une partie des fibres solubles, ce qui a abouti à un perméat clair et limpide correspondant à la demande du consommateur.

\subsubsection{Microstructure du colmatage}

Pour avoir une idée sur la nature et la structure du colmatage formé au contact immédiat de la couche active de la membrane utilisée pour la microfiltration, nous avons effectué des observations au microscope à balayage électronique après différents temps de contact, en statique, entre la membrane et la solution brute issue de la pâte de datte ou entre la membrane et la solution préalablement clarifiée par un mélange d'enzymes (figure 5).

La solution préalablement clarifiée laisse un dépôt édifié, formé d'un réseau d'agrégats sphériques et linéaires. Cependant, le réseau obtenu après traitement de la solution brute non prétraitée est plus dense et les agrégats qui le constituent sont moins individualisés et présentent de fins liens filamenteux entre un certain nombre de particules. Cette structure obtenue en statique serait comparable à celle édifiée en dynamique après la mise en place de l'état stationnaire. 


\section{Conclusion}

La composition chimique des sous-produits de dattes de la variété Deglet Nour est riche en sucres, protéines, minéraux et fibres.

La boisson brute qui a été extraite à partir d'écarts de triage de dattes est faiblement acide, riche en fraction glucidique et minérale, mais trouble, ce qui nous a conduit à la clarifier.

La microfiltration a montré que les sucres simples, les éléments minéraux et les protéines solubles traversent librement la lumière des pores de la membrane utilisée lors du procédé et se retrouvent dans le perméat. Les polysaccharides et des fibres insolubles ont été en revanche retenus par la membrane comme en témoigne la réduction de la turbidité du perméat.

Un traitement enzymatique préalable de la boisson brute a induit une réduction supplémentaire de la turbidité et de la viscosité du fait de la dégradation des fibres et de l'apparition d'acide galacturonique et de sucres simples. Au cours de cette hydrolyse, un effet synergique a été remarqué entre l'action des pectinases et celle des cellulases ; il s'est traduit par une diminution plus importante de la viscosité et de la turbidité.

L'effet conjugué du mélange pectinases + cellulases est donc plus efficace que l'un ou l'autre de ces deux enzymes utilisé seul. Cette efficacité est illustrée par un flux de perméats deux fois plus important que celui observé pour une boisson non prétraitée par ces enzymes et par une fraction glucidique et protéique nettement supérieure. Dans les boissons de datte clarifiées, cela a induit une augmentation de la matière sèche et du contenu en solides solubles.

\section{Références}

[1] Booji I., Piompo G., Risterucci J.M., Thomas D., Ferry M., Sugar and free amino acid composition of five cultures of dates from offshoot or vitroplants in open fields, Agric. Food Chem. 41 (1993) 1553-1557.

[2] Fayadh J.M., Al-Showiman S.S., Chemical composition of date palm (Phoenix dactylifera L.), Chem. Sos. Pak. 12 (1990) 84-103.
[3] Khatchadourian H.A., Sawaya W.N., Khalil J., Mashadi A.S., Processing of five major Saudi Arabian date varieties into dates butter and dates in syrup, Date Palm 1 (1983) 103-119.

[4] Yousif A.K., Abou Ali M., Abou Idreese A., Processing, evaluation and storability of date jelly, Food Sci. Technol. 27 (1990) 264267.

[5] Al-Hooti S., Jiuan S., Quabazard H., Studies on the physico-chemical characteristics of date fruits of five UAE cultivars at different stages of maturity, Arab. Gulf J. 13 (1995) 553-569.

[6] Yousif A.K., Alghamdi A.S., Hamad A., Mustafa A.I., Processing and evaluation of a date juice-milk drink, Egypt. J. Dairy Sci. 24 (1996) 277-288.

[7] Hobani A.I., Rheological behaviour of datewater concentrates, Food Eng. 36 (1998) 349-357.

[8] Al Obaidi Z.S., Berry D.R., Saling up of citric acid fermentation using fermenters, Date Palm 2 (1982) 19-33.

[9] Nacib N., Nacib A., Bourdant J., Use of waste products in the fermentative formation of Baker's yeast biomass by Saccharomyces cervisiae, Biores. Technol. 60 (1997) 67-71.

[10] Nacib N., Nacib A., Mostefa G., Larbi L., Adimi L., Remmal M., Bourdant J., Use of date products in production of thermophilic dairy starters strain Streptococcus thermophilus, Biores. Technol. 67 (1999) 291295

[11] Abou Zied A.A., Baghlef A.O., Utilization of date seeds and cheese whey in production of citric acid by Candida lipolytica, Agric. Wastes 8 (1983) 131-142.

[12] Abou Zied A.A., Abderrahman N., Baghlef A.O., The formation of oxytetracycline in date-coat medium, Biores. Technol. 37 (1991) 179-184.

[13] Jraidi Z., Mahjoub A., Ferjani T., Essai d'élaboration de confiture de dattes, Rev. Inat (1990) 191-197.

[14] Vaillant F., Cisse M., Chaverri M., Perez A., Dornier M., Viquez F., Dhuique-Mayer C., Clarification and concentration of melon juice using membrane processes, Innov. Food Sci. Emerg. Technol. 6 (2005) 213-220.

[15] Yu J., Lencki R.W., Effect of enzyme treatments on the fouling behavior of apple juice 
during microfiltration, J. Food Eng. 63 (2004) 413-423.

[16] Decloux M., Prothon F., Les séparations par membrane dans les procédés de l'industrie alimentaire, Tec. Doc., Lavoisier, Paris, France, 1998.

[17] Attia H., Bennasar M., Tarodo De La Fuente B., Ultrafiltration sur membrane minérale de laits acidifiés à divers $\mathrm{pH}$ par voie biologique ou chimique et de coagulum lactique, Lait 68 (1) (1988) 13-32.

[18] Anon., Official methods of analyses, Assoc. Off. Anal. Chem., AOAC, Wash., USA, 1997.

[19] Bradford M.M., A rapid and sensitive method for the quantification of microgram quantities of protein utilising the principle of protein-dye binding, Anal. Biochem. 72 (1976) 248-254.

[20] Miller G.L., Use of dinitrosalycilic acid reagent for determination of reducing sugar, Anal. Chem. 31 (1959) 426-428.

[21] Prosky L., Asp N.-G., Scheweizer T.F., De Vries J.W., Furada I., Determination of insoluble and soluble, and total dietary fibre in foods and food products: interlaboratory study, J. Assoc. Off. Anal. Chem. 71 (5) (1988) 1017-1023.

[22] Vétier C., Étude du colmatage d'une membrane minérale de microfiltration par microscopie électronique à balayage et analyses physico-chimiques : application au lait, Univ. Sci. Tech. Languedoc, Thèse, Montpellier, France, 1986, $35 \mathrm{p}$.

[23] Attia H., Bennasar M., Lagaude A., Hugodo B., Rouviere J., Tarodo De La Fuente B., Ultrafiltration with microfiltration membrane of acid skimmed and fat-enriched milk coagula: hydrodynamic, microscope and rheological approaches, Dairy Res. 60 (1993) 161-174.

[24] Al-Hooti S.N., Sidhu J.S., Al-Saqer J.M., AlOthman A., Chemical composition and quality of date syrup as affected by pectinase/ cellulase enzyme treatment, Food Chem. 79 (2002) 215-220.

[25] Ahmed I.S.A., Al-Gharibi K.N., Daar A.S., Kabir S., The composition and properties of date proteins, Food Chem. 53 (1995) 441446.
[26] Bouabidi H., Reyens M., Roussi, M.B., Critères de caractérisation des fruits de quelques cultivars de palmiers dattiers (Phoenix dactylifera L.) du sud tunisien, Ann. Inrat (1996) 69-78.

[27] Vandercook C.E., Hasegawa S., Maier V.P., Quality and nutritive value of dates as influenced by their chemical composition, Date Grow. Inst. Rep. 54 (1997) 3-11.

[28] Barreveld W.H., Date palm products, Bull. Serv. FAO, 101, Roma, Italy, 1993.

[29] Cassano A., Donato L., Drioli E., Ultrafiltration of kiwifruit juice: operating parameters, juice quality and membrane fouling, J. Food Eng. (2006) (in press).

[30] Borneman Z., Gokmen V., Nijhuis H.H., Selective removal of polyphenols and brown colour in apple juices using PES/PVP membranes in a single-ultrafiltration process, Membr. Sci., 14 (1997) 191-197.

[31] Sin H.N., Yusof S., Sheikh Abdul Hamid N., Abd. Rahman R., Optimization of enzymatic clarification of sapodilla juice using response surface methodology, J. Food Eng. 73 (2006) 313-319.

[32] Vaillant F., Millan A., Dornier M., Decloux M., Reynes M., Strategy for economical optimisation of the clarification of pulpy fruit juices using crossflow microfiltration, J. Food Eng. 48 (2001) 83-90.

[33] Alvarez S., Alvarez A., Riera F.A., Coca J., Influence of depectinization on apple juice ultrafiltration, Colloid. Surf. A: Physicochem. Eng. Asp. 138 (1998) 377-382.

[34] Campos D.C.P., Santos A.S., Wolkoff D.B., Matta V.M., Cabral L.M.C., Couri S., Cashew apple juice stabilization by microfiltration, Desalin. 148 (2002) 61-65.

[35] Vladisavljevi G.T., Vukosavljevi P., Bukvi B., Permeate flux and fouling resistance in ultrafiltration of depectinized apple juice using ceramic membranes, J. Food Eng. 60 (2003) 241-247.

[36] Jean F., Broussous P., Ferrari G., Cabanis, J.C., Mise au point d'un procédé de clarification des jus de fruits de kiwi, Ind. Agroaliment. (1991) 343-348. 


\section{Elaboración de una bebida a partir de los subproductos de dátiles: clarificación mediante tratamiento enzimático y microfiltración.}

Resumen - Introducción. En Túnez, gran productor de dátiles, los subproductos de dátiles permanecen sin estar valorizadas, a pesar de que dispongan de un valor nutricional comparable a la del fruto de gran valor comercial. Nuestro estudio tuvo como objetivo el testeo de una vía de valorización basada en la extracción, a partir de una pasta de dátil, de una bebida de dátil aclarada. Material y métodos. El esquema tecnológico del procedimiento de extracción de la pulpa se inspiró de un procedimiento tradicional. Se trató una solución bruta, mediante vía enzimática (pectinazas, celulazas) y mediante microfiltración tangencial, obtenida de la dilución de pasta de dátiles. Se determinaron la composición química, las características fisicoquímicas, así como las características reológicas de la bebida aclarada obtenida. Resultados y discusión. La solución bruta extraída de los dátiles después de la trituración, dilución y filtración tiene un alto contenido en azúcar. El acoplamiento de los tratamientos enzimáticos y de microfiltración condujo a la elaboración de una bebida aclarada, moderadamente ácida, presentando una viscosidad y una turbidez relativamente bajas y manifestando características de liquidez comparables a las de las bebidas y zumos de frutas conocidos. Conclusión. Las separaciones de la selección de dátiles pueden constituir un subproducto apto para ser transformado, mediante procedimientos habituales, en una bebida agradable y comercial.

Túnez/Pboenix dactylifera / dátil / subproductos / aprovechamiento de desechos / transformación (de productos) 